\title{
Radiation and Spontaneous Annealing of Radiation-sensitive Field-effect Transistors with Gate Oxide Thicknesses of 400 and $1000 \mathrm{~nm}$
}

\author{
Goran S. Ristic,,$^{*}$ Marko S. Andjelković, ${ }^{2}$ Russell Duane, ${ }^{3}$ \\ Alberto J. Palma, ${ }^{4}$ and Aleksandar B. Jakšić ${ }^{3}$ \\ ${ }^{1}$ Faculty of Electronic Engineering, University of Niš, P.O. Box 73, 18000 Niš, Serbia \\ ${ }^{2} \mathrm{IHP} \mathrm{GmbH}$ - Innovations for High Performance Microelectronics (IHP), \\ Im Technologiepark 25, 15236 Frankfurt (Oder), Germany \\ ${ }^{3}$ Tyndall National Institute, University College Cork, Lee Maltings Complex Dyke Parade, Cork, T12 R5CP, Ireland \\ ${ }^{4}$ University of Granada, Department of Electronics and Computer Science, \\ Avenida de la Fuente Nueva S/N CP:18071 Granada, Spain
}

(Received May 6, 2021; accepted May 21, 2021)

Keywords: RADFETs, radiation dosimeters, radiation, annealing, fading

We investigated the influence of gamma radiation of $50 \mathrm{~Gy}\left(\mathrm{H}_{2} \mathrm{O}\right)$ on radiation-sensitive p-channel metal-oxide-semiconductor field-effect transistors with an Al gate (RADFETs) with gate oxide thicknesses of 400 and $1000 \mathrm{~nm}$ and gate voltages of 0 and $5 \mathrm{~V}$. The obtained results showed that the sensitivity $S$ at a given gate voltage increases with the square of the gate oxide thickness. After irradiation (IR), spontaneous annealing (SA) was performed at room temperature without voltage at the gate. We present the behaviors of fixed traps and switching traps, determined by the midgap technique, and that of fast switching traps, determined by the charge-pumping technique, during IR and SA. A very important characteristic of dosimetric transistors is fading, which represents the recovery of the threshold voltage of the irradiated RADFETs during SA. The maximum fading is about $15 \%$ after $9100 \mathrm{~h}$, except for the RADFETs with a gate oxide thickness of $1000 \mathrm{~nm}$ and a gate voltage of $5 \mathrm{~V}$, for which it is about $30 \%$. A fitting equation for fading was proposed, which fitted the experimental fading values very well.

\section{Introduction}

Radiation-sensitive field-effect transistors (known as RADFETs or pMOS dosimeters) can be used as radiation dosimeters for applications such as space, the nuclear industry, and radiotherapy. ${ }^{(1-7)}$ RADFETs have a small sensor element of less than $1 \times 1 \mathrm{~mm}^{2}$ and allow realtime dose measurement in vivo, which is especially important for radiotherapy. A RADFET can be used as a wireless dosimeter, permanently implanted in vivo. ${ }^{(8)}$ It is encapsulated in hermetically sealed glass cylinders and used in an unbiased mode during irradiation. The transistor is powered by radio frequency telemetry for dose measurements, avoiding the need for a power supply within the capsule.

*Corresponding author: e-mail: goran.ristic@elfak.ni.ac.rs https://doi.org/10.18494/SAM.2021.3425 
The advantages of RADFETs over other dosimetric systems are nondestructive reading of dosimetric information, very low power consumption, full compatibility with microprocessors, wide dose range, and low price (especially if the cost of the reading system is considered). However, they also have the disadvantages of low sensitivity (starting from about $1 \mathrm{rad}$ ), low reusability, and high fading (up to 50\%). Thus, it is very important to increase their sensitivity and reduce fading. The sensitivity can be increased by increasing the thickness of the oxide, whereas it remains unclear how to reduce fading.

The pMOS dosimeter concept is based on the measurement of threshold voltage shifts, $\Delta V_{T}$, caused by both radiation-induced fixed traps (FTs) in the gate oxide and switching traps (STs) near and at the oxide/substrate interface, and converting $\Delta V_{T}$ into the absorbed dose $D$. Both types of traps increase $\Delta V_{T}$ in pMOS transistors, while FTs increase $\Delta V_{T}$ but STs decrease $\Delta V_{T}$ in nMOS transistors. Therefore, pMOS transistors are used as radiation dosimeters instead of nMOS transistors.

To increase the sensitivity of pMOS dosimeters, transistors with a thick gate oxide (400 and $1000 \mathrm{~nm}$ ) were used in this work. Given the importance of traps in the oxide and at the oxide/ substrate interface for sensitivity and fading, the behaviors of their densities during irradiation (IR) and spontaneous annealing (SA) were investigated.

\section{Experimental Details}

Specially designed, radiation-sensitive $p$-channel metal-oxide-semiconductor field-effect transistors with an Al gate (RADFETs) manufactured at Tyndall National Institute, Cork, Ireland, having gate oxide thicknesses of $t_{o x 1}=400 \mathrm{~nm}$ and $t_{o x 2}=1000 \mathrm{~nm}$, were used. ${ }^{(9)}$ The chips of these RADFETs typically contain four transistors, but only the first transistor on the chip (transistor \#T1) was tested.

The RADFETs were irradiated with an ionizing ${ }^{60} \mathrm{Co}$ source to an absorbed dose of $D=50$ $\mathrm{Gy}\left(\mathrm{H}_{2} \mathrm{O}\right)$. During the radiation, the voltages at the gate were $V_{G, i}=0$ and $5 \mathrm{~V}$. After IR, the RADFETs were annealed by SA for $9100 \mathrm{~h}$ at room temperature without a gate voltage $\left(V_{G, a}=0\right.$ $\mathrm{V})$. In cases without a gate voltage during IR and SA $\left(V_{G, i}=0 \mathrm{~V}\right.$ and $\left.V_{G, a}=0 \mathrm{~V}\right)$, all pins were short-circuited and grounded. For pMOSFETs with an Al gate, such as the RADFETs used in this study, for the zero-bias mode (no gate voltage), there is a small positive gate voltage of $V_{w f}=$ $0.33 \mathrm{~V}$ due to the difference in the work function between the Al gate and the $n$-type silicon substrate, which results in a small positive external electric field in the gate oxide. ${ }^{(10)}$

The electrical characteristics of the RADFETs during IR and SA were measured using an automatic system guided by a program written in C\# (see Ref. 11 for more details). The threshold voltage of the RADFETs before IR, $V_{T 0}$ (given in Table 1), during IR, $V_{T, i r r}$, and during SA, $V_{T, s a}$, were determined from the electrical transfer characteristics under saturation, as the intersection between the gate voltage $V_{G}$ axis and the extrapolated linear region of the $\left(I_{D}\right)^{1 / 2}-V_{G}$ curve, where $I_{D}$ is the drain current, using the least squares method performed in the Octave 6.2.0 program. . $^{(2)}$

The threshold voltage shifts during IR and SA are

$$
\Delta V_{T, i r r}=V_{T, i r r}-V_{T 0}, \Delta V_{T, s a}=V_{T, s a}-V_{T 0} .
$$


Table 1

RADFET characteristics, experimental conditions, and sensitivity $S$.

\begin{tabular}{lccccl}
\hline$t_{o x}(\mathrm{~nm})$ & Label of used RADFET & $V_{G, i}(\mathrm{~V})$ & $V_{G, a}(\mathrm{~V})$ & $V_{T 0}(\mathrm{~V})$ & \multicolumn{1}{c}{$S(\mathrm{~V} / \mathrm{Gy})$} \\
\hline \multirow{2}{*}{400} & TYNDALL 04 P-3460 W4 3607 015 & 0 & 0 & 3.46 & 0.17778 \\
& TYNDALL 04 P-3460 W4 3607 018 & 5 & 0 & 3.49 & 0.0287 \\
\hline \multirow{2}{*}{1000} & TYNDALL 04 X1370 W4 3109 045 & 0 & 0 & 1.96 & 0.288 \\
& TYNDALL 04 X1370 W4 3109 046 & 5 & 0 & 2.16 & 0.04592 \\
\hline
\end{tabular}

The midgap-subthreshold technique (MGT) was used, which determines the components of the threshold voltage shifts induced by FTs, $\Delta V_{f t}$, and STs, $\Delta V_{s t}{ }^{(13)}$

The relationship between these components and the threshold voltage shift $\Delta V_{T}$ during IR and SA is

$$
\Delta V_{T}=\Delta V_{f t}+\Delta V_{s t}
$$

The areal densities of FTs, $\Delta N_{f t}\left(\mathrm{~cm}^{-2}\right)$, and STs, $\Delta N_{s t}\left(\mathrm{~cm}^{-2}\right)$ for pMOSFETs are ${ }^{(12)}$

$$
\Delta N_{f t}=\frac{C_{o x}}{e} \Delta V_{f t}, \Delta N_{s t}=\frac{C_{o x}}{e} \Delta V_{s t},
$$

where $C_{o x}=\varepsilon_{o x} / t_{o x}$ is the gate oxide capacitance per unit area, $\varepsilon_{o x}=3.45 \times 10^{-13} \mathrm{~F} / \mathrm{cm}$ is the permittivity of silicon dioxide, and $e$ is the absolute value of the electron charge.

The STs contain slow switching traps (SSTs) near the oxide/substrate interface and fast switching traps (FSTs) exactly at the interface, and $\Delta N_{s t}$ can be expressed as ${ }^{(10)}$

$$
\Delta N_{s t}=\Delta N_{s s t}+\Delta N_{f s t}
$$

where $\Delta N_{s s t}$ and $\Delta N_{f s t}$ are the areal densities of SSTs and FSTs, respectively.

Because the used RADFETs have four pins [gate, drain, source, and substrate (bulk) pins], we were able to apply the charge-pumping technique (CPT). As a much faster technique than the MGT (a few Hz for the MGT compared with a few MHz for the CPT), the CPT only determines the density of FSTs. More details of the CPT can be found in Refs. 12 and 14.

\section{Results and Discussion}

$\Delta V_{T}$ during IR and SA is shown in Fig. 1. Although $\Delta V_{T}$ is not generally linear and saturates with increasing $D{ }^{(10)}$ the linear function $\Delta V_{T}=S \cdot D$ can be employed for small doses, where $S$ is the sensitivity. Using this function, we fitted $\Delta V_{T}$ with $D$ and obtained a very good agreement (the correlation coefficients $r^{2}$ were greater than 0.99). The values of $S$ are shown in Table 1. The largest drop in $\Delta V_{T}$ during SA is for the RADFET with $t_{o x}=1000 \mathrm{~nm}$, whereas the other RADFETs show significantly smaller changes. 


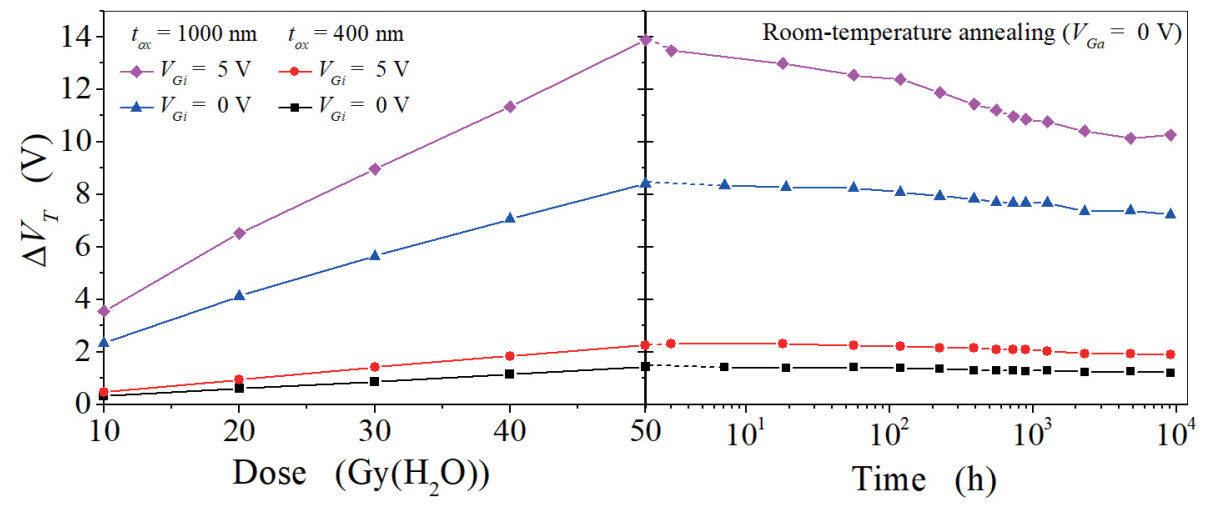

Fig. 1. (Color online) Threshold voltage shift during IR and SA.

It can be seen from Table 1 that the ratio between the sensitivities for the two oxide thicknesses at the given gate voltage is $S_{1000} / S_{400} \approx 6.25=2.5^{2}$, whereas the ratio between the thicknesses is $\left(t_{o x 2} / t_{o x 1}\right)^{2}=(1000 / 400)^{2}=2.5^{2}$. This shows that the sensitivity increases with the square of the gate oxide thickness, $t_{o x}\left(S \sim t_{o x}{ }^{2}\right)$.

Figures 2 and 3 respectively display the behaviors of the areal densities of FTs $\left(\Delta N_{f t}\right)$ and STs $\left(\Delta N_{s t}\right)$ during IR and SA obtained by the MGT. The highest density of the FTs is for $t_{o x}=1000$ $\mathrm{nm}$ and $V_{G, i}=5 \mathrm{~V}$, and the lowest density is for $t_{o x}=400 \mathrm{~nm}$ and $V_{G, i}=0 \mathrm{~V}$.

By comparing these two types of RADFETs, it is expected that during radiation, more traps will be created in the thicker oxide, which has a larger volume of oxide (the same surface area and greater thickness). Also, a higher external positive electric field, $E_{\text {ext }}$, leads to a higher density of FTs by reducing the probability of recombination of electrons and holes formed during radiation and by increasing the transport of holes to the oxide-semiconductor interface. Near the interface, there are energetically deeper trap centers of the holes. ${ }^{(12)}$

On the other hand, the density of FSTs $\left(\Delta N_{f s}\right)$ should not depend on $t_{o x}$ and should only depend on the oxide surface area. Since both oxides have the same surface area, they should have the same $\Delta N_{f s t}$. However, $\Delta N_{f s t}$ also depends on the $E_{\text {ext }}$, forcing the holes responsible for creating FSTs to the $\mathrm{SiO}_{2} / \mathrm{Si}$ interface. The density of SSTs $\left(\Delta N_{s s t}\right)$ should depend on $t_{\text {ox }}$ and $E_{\text {ext }}$, since SSTs are located in the oxide, near the $\mathrm{SiO}_{2} / \mathrm{Si}$ interface. Figure 3 confirms that $\Delta N_{s t}$ depends on $t_{o x}$ and $E_{e x t}$.

Figure 4 shows the areal densities of FTs and STs for the RADFET with $t_{o x}=1000 \mathrm{~nm}$ and $V_{G, i}=5 \mathrm{~V}$. These densities show typical behavior. The values of $\Delta N_{f t}$ during IR are more than twice those of $\Delta N_{s t}$. During SA, $\Delta N_{f t}$ decreases markedly, whereas $\Delta N_{s t}$ increases slightly. Namely, whereas FTs recover to a significant extent, STs are being created.

The CPT was also applied. As mentioned above, the CPT is much faster than the MGT, which registers all STs (SSTs and FSTs), whereas the CPT registers only FSTs. However, these techniques cannot be quantitatively compared. Namely, the two techniques scan different parts of the Si bandgap: the lower half (MGT) versus the central part (CPT). As interface traps have a U-shaped distribution along the edges of the bandgap and the CPT cannot reach that part, this can lead to lower CPT values. Figure 5 shows that the qualitative behaviors of $\Delta N_{s t}$ (MGT) and 


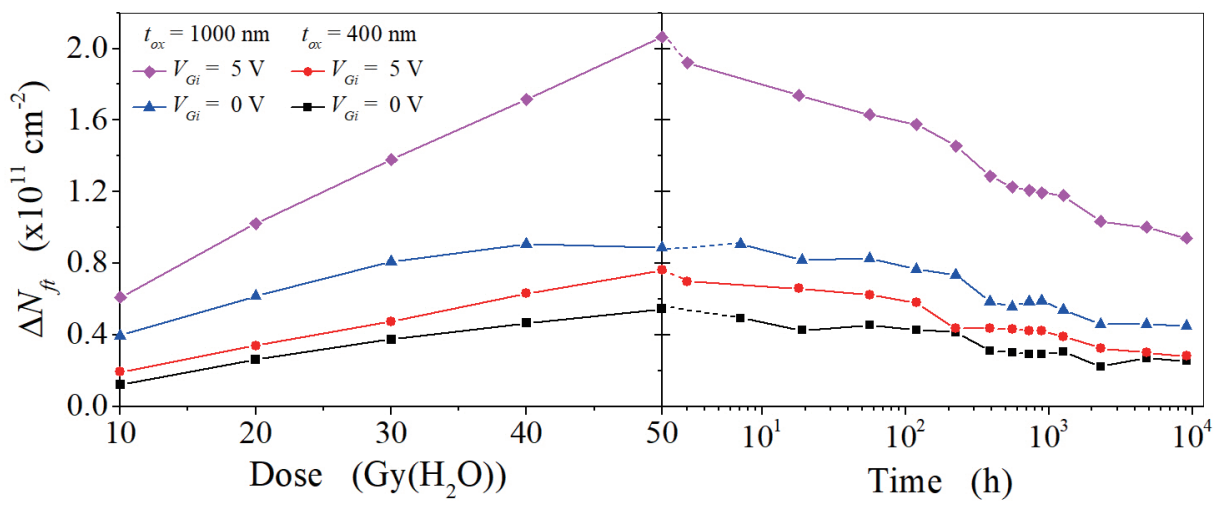

Fig. 2. (Color online) Areal density of FTs during IR and SA.

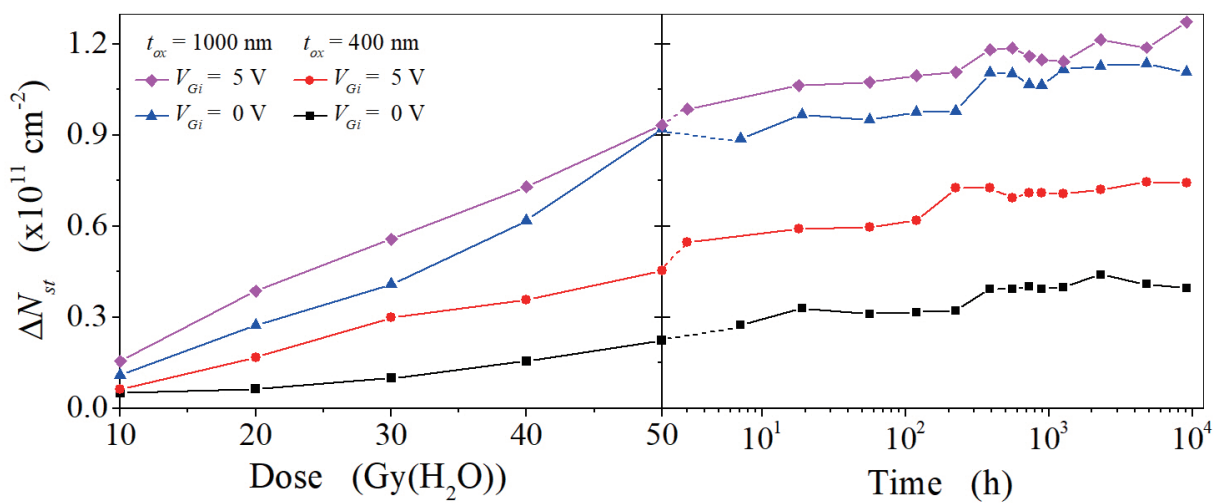

Fig. 3. (Color online) Areal density of STs during IR and SA.

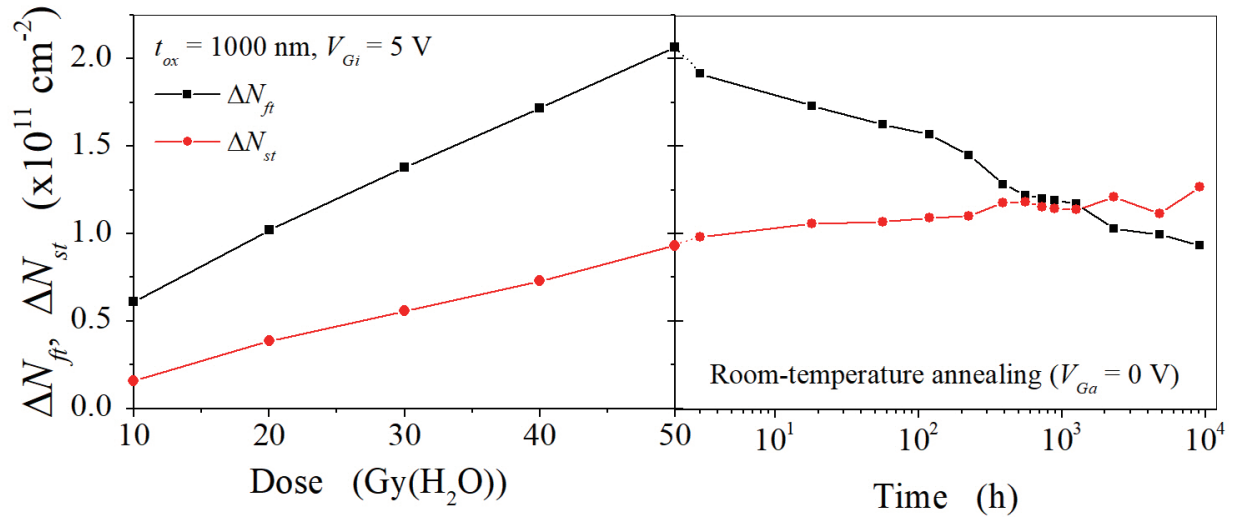

Fig. 4. (Color online) Areal densities of FTs and STs during IR and SA.

$\Delta N_{f s t}(\mathrm{CPT})$ are different. The STs are created during IR and SA, with more STs created during IR. The density of FSTs saturates during IR and decreases slightly during SA.

SA can be more easily analyzed by fading, which represents the recovery of the threshold voltage of irradiated RADFETs during SA, defined as ${ }^{(15)}$ 


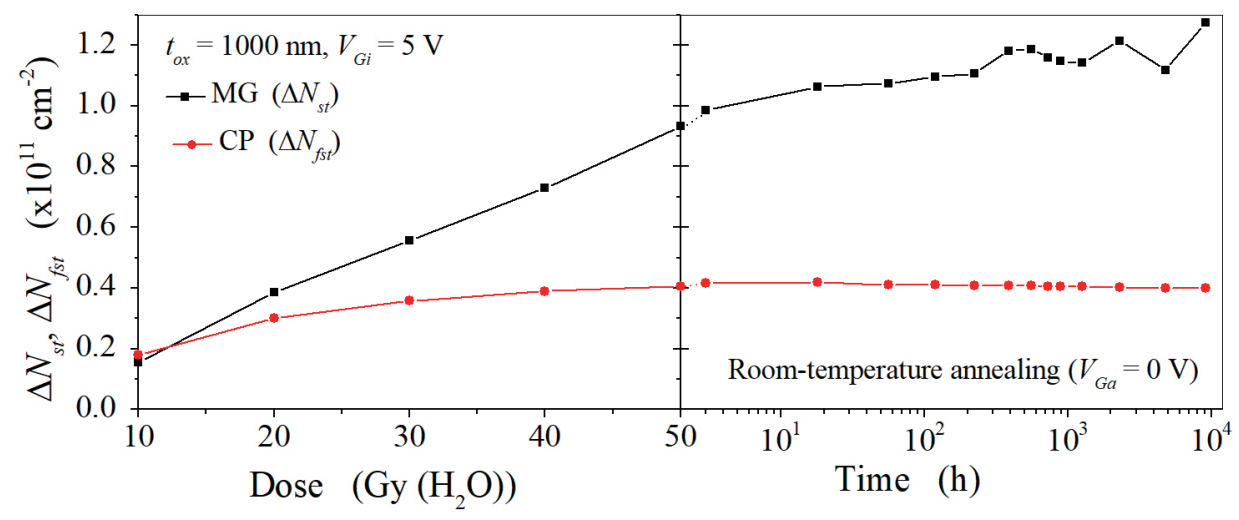

Fig. 5. (Color online) Densities of FTs, obtained by MGT, and of FFTs, obtained by CPT.

$$
f=\frac{V_{T, i r r}(50 \mathrm{~Gy})-V_{T, s a}(t)}{V_{T, i r r}(50 \mathrm{~Gy})-V_{T 0}}=\frac{\Delta V_{T, i r r}(50 \mathrm{~Gy})-\Delta V_{T, s a}(t)}{\Delta V_{T, i r r}(50 \mathrm{~Gy})},
$$

where $V_{T, i r r}(50 \mathrm{~Gy})$ is the threshold voltage after IR, i.e., at $D=50 \mathrm{~Gy}, V_{T, s a}(t)$ is the threshold voltage during SA, and $V_{T 0}$ is the transistor threshold voltage before IR.

On the basis of the research presented in this paper, the following function can fit the fading:

$$
f=f_{\text {sat }}-\frac{f_{\text {sat }}}{1+b t^{c}}
$$

where $t$ is the annealing time, $f_{\text {sat }}$ is the saturation value of the fading, and $b$ and $c$ are positive constants. The fading values obtained by Eq. (5) and their fit by Eq. (6) are presented in Fig. 6. Good fitting can be observed. Table 2 shows the parameters used in the fitting, as well as the fading values at the end of SA $(9100 \mathrm{~h})$.

All the presented results were obtained by measuring the electrical characteristics using a constant voltage at the drain, $V_{D}$, which is higher than the voltage at the gate, $V_{G}\left(V_{D}=\right.$ const., $\left.V_{D}>V_{G}\right) . V_{G}$ was changed from zero to a certain negative value and the drain current $I_{D}$ was measured. However, for faster measurement of the electrical characteristics of the RADFETs, the drain and the gate, as well as the source and bulk should be shortcutted $\left(V_{D}=V_{G}\right.$ and $V_{S}=V_{B}$; so-called reader-circuit (RC) configuration). $I_{D}$ is forced and $V_{G}$ is measured. In this way, it is much faster to measure the electrical characteristics of a transistor. We used both methods to measure the electrical characteristics.

Figure 7(a) shows the electrical subthreshold characteristics of the RADFET with $t_{o x}=400$ $\mathrm{nm}$ and $V_{G, i}=0 \mathrm{~V}$ for absorbed doses of 20 and $50 \mathrm{~Gy}\left(\mathrm{H}_{2} \mathrm{O}\right)$. The $\mathrm{RC}$ configuration cannot reach a low current level. Although the characteristics between $I_{D}=5 \times 10^{-8}$ and $5 \times 10^{-7} \mathrm{~A}$, which are used in the MGT, visually match, there are small differences between them, which result in differences in the obtained densities $\Delta N_{f t}$ and $\Delta N_{s t}$ [Fig. 7(b)]. However, these differences are less than $10 \%$, and the behaviors of $\Delta N_{f t}$ and $\Delta N_{s t}$ are the same. Otherwise, these differences do not affect the threshold voltages, which are equal for both cases. 


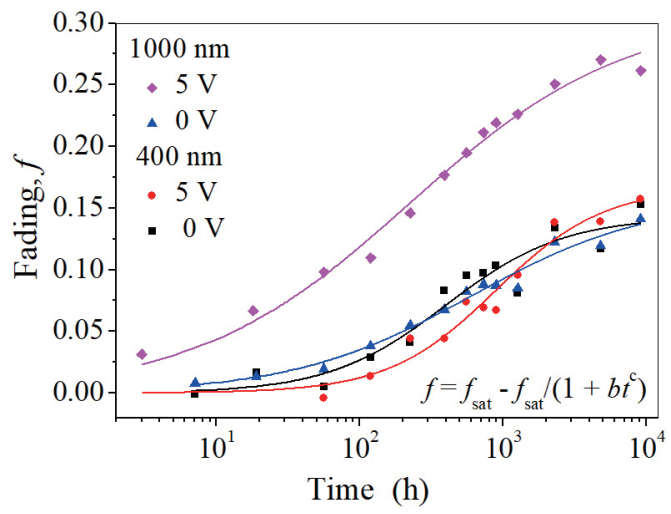

Fig. 6. (Color online) Fading fitted by Eq. (6).

Table 2

Fitting parameters used in Fig. 6.

\begin{tabular}{lccclc}
\hline$t_{\text {ox }}(\mathrm{nm})$ & $V_{G, i}(\mathrm{~V})$ & $f @ 9100 \mathrm{~h}$ & \multicolumn{1}{c}{$f_{\text {sat }}$} & \multicolumn{1}{c}{$b$} & $c$ \\
\multirow{2}{*}{400} & 0 & 0.1532 & 0.1425 & 0.0017456 & 1.06049 \\
& 5 & 0.1576 & 0.1666 & 0.000393 & 1.15315 \\
\hline \multirow{2}{*}{1000} & 0 & 0.1411 & 0.1571 & 0.0115898 & 0.69709 \\
& 5 & 0.2615 & 0.3079 & 0.0435784 & 0.57903
\end{tabular}

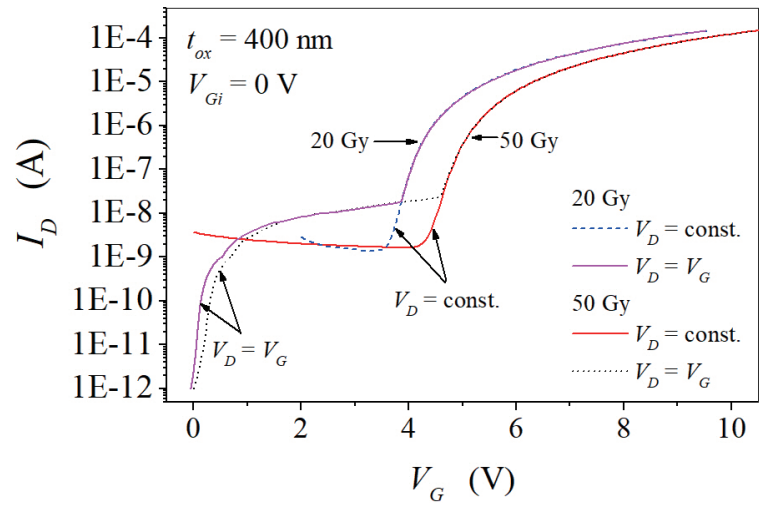

(a)

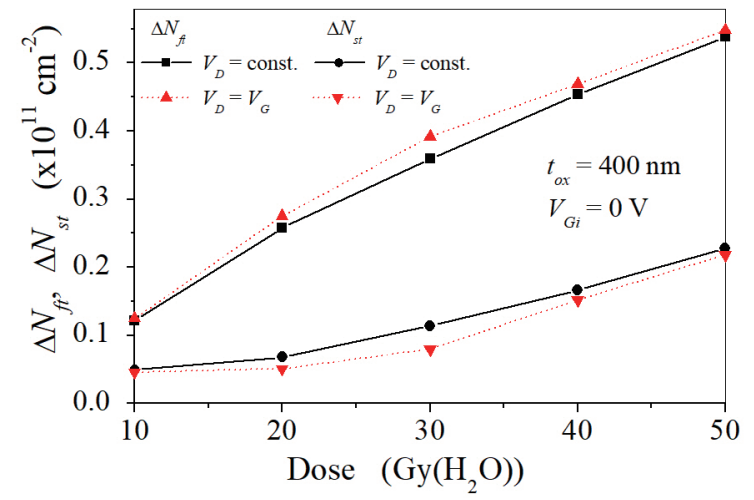

(b)

Fig. 7. (Color online) (a) Electrical subthreshold characteristics and (b) densities $\Delta N_{f t}$ and $\Delta N_{s t}$ during IR.

\section{Conclusions}

Experimental results have shown that the sensitivity of RADFETs increases with the square of the oxide thickness at a given voltage. The values of $\Delta N_{f t}$ during IR are more than twice those of $\Delta N_{s t}$. During SA, $\Delta N_{f t}$ decreases markedly, whereas $\Delta N_{s t}$ increases slightly. The behaviors of STs and FSTs are different during IR and SA. As the density of STs increases, the density of FSTs saturates and decreases slightly. The proposed fading function fits the experimental values 
very well. It has also been shown that the electrical characteristics obtained by the RC configuration can be used to determine the density of traps in the oxide and at the interface.

\section{Acknowledgments}

This work was supported in part by the European Union's Horizon 2020 research and innovation programme (Grant No. 857558) and the Ministry of Education, Science and Technology Development of the Republic of Serbia (Project No. 43011).

\section{References}

1 M. J. Butson, T. Cheung, and P. K. N. Yu: Appl. Radiat. Isot. 62 (2005) 631. https://doi.org/10.1016/j. apradiso.2004.09.001

2 L. Frohlich, K. Casarin, E. Quai, A. Holmes-Siedle, M. Severgnini, and R. Vidimari: Nucl. Instrum. Methods Phys. Res., Sect. A 703 (2013) 70. https://doi.org/10.1016/j.nima.2012.11.021

3 P. Gonçalves, A. Keating, A. Trindade, P. Rodrigues, M. Ferreira, P. Assis, M. Muschitiello, B. Nickson, and C. Poivey: IEEE Trans. Nucl. Sci. 61 (2014) 1439. https://doi.org/10.1109/TNS.2014.2321477

4 I. Mateu, M. Glaser, G. Gorine, M. Moll, G. Pezzullo, and F. Ravotti: IEEE Trans. Nucl. Sci. 65 (2018) 1700. https://doi.org/10.1109/TNS.2017.2784684

5 M. Kulhar, K. Dhoot, and A. Pandya: IEEE Trans. Nucl. Sci. 66 (2019) 2220. https://doi.org/10.1109/ TNS.2019.2942955

6 S. Jain, S. G. Surya, P. K. Suggisetti, A. Gupta, and V. R. Rao: IEEE Sens. J. 19 (2019) 4428. https://doi. org/10.1109/JSEN.2019.2901810

7 A. B. Rosenfeld, G. Biasi, M. Petasecca, M. L. F. Lerch, G. Villani, and V. Feygelman: Phys. Med. Biol. 65 (2020) TR01. https://doi.org/10.1088/1361-6560/aba163

8 A. S. Beddar, M. Salehpour, T. M. Briere, H. Hamidian, and M. T. Gillin: Phys. Med. Biol. 50 (2004) 141. https://doi.org/10.1088/0031-9155/50/1/011

9 M. S. Andjelković, G. S. Ristić, and A. B. Jakšić: Meas. Sci. Technol. 26 (2015) 025004. https://doi. org/10.1088/0957-0233/26/2/025004

10 G. S. Ristic, M. S. Andjelkovic, and A. B. Jaksic: Appl. Radiat. Isot. 102 (2015) 29. https://doi.org/10.1016/j. apradiso.2015.04.009.

11 G. S. Ristić, N. D. Vasović, M. Kovačević, and A. B. Jakšić: Nucl. Instrum. Methods Phys. Res., Sect. B 269 (2011) 2703. https://doi.org/10.1016/j.nimb.2011.08.015

12 G. S. Ristić: J. Phys. D: Appl. Phys 41 (2008) 023001. https://doi.org/10.1088/0022-3727/41/2/023001

13 P. J. McWhorter and P. S. Winokur: Appl. Phys. Lett. 48 (1986) 133. https://doi.org/10.1063/1.96974

14 G. Groeseneken, H. E. Maes, N. Beltran, and R. F. De Keersmaecker: IEEE Trans. Electron Devices ED-31 (1984) 42. https://doi.org/10.1109/T-ED.1984.21472

15 G. S. Ristić: J. Phys. D: Appl. Phys. 42 (2009) 135101. https://doi.org/10.1088/0022-3727/42/13/135101 